\title{
Regular Sparse Crossbar Concentrators
}

Weiming Guo, Member, IEEE, and A. Yavuz Oruç, Senior Member, IEEE

\begin{abstract}
A bipartite concentrator is a single stage sparse crossbar switching device that can connect any $m$ of its $n \geq m$ inputs to its $m$ outputs, possibly without the ability to distinguish their order. Fat-andslim crossbars were introduced recently to show that bipartite concentrators can be constructed with a minimum number of crosspoints for any number of inputs and outputs. We generalize these graphs to obtain bipartite concentrators with nearly a fixed fanout without altering their $(n-m+1) m$ crosspoint complexity. We also present an $O(\log n)$-time algorithm to route arbitrary concentration assignments on this new family of fat-and-slim crossbars.
\end{abstract}

Index Terms-Bipartite graph, concentrator, crosspoint complexity, regular sparse crossbar.

\section{INTRODUCTION}

A number of models have been introduced to deal with concentration operations in multiprocessor systems. The most easily understood among these models is a concentrator switch. An $(n, m)$ concentrator is a switching device with $n$ inputs and $m$ outputs that permits nonoverlapping paths between any $m$ of the $n$ inputs and the $m$ outputs. These devices have been studied extensively in the interconnection network literature, and key theoretical results point out that $(n, m)$-concentrators can be constructed with $O(n)$ crosspoints and $O(\log n)$ delay [10], [2], [1]. Despite these findings, many of the explicit concentrator designs reported in the literature use $O(n \log n)$ crosspoints, and typically rely on butterfly graphs [4], and adaptive binary sorting networks [8].

In this paper, we present a number of new sparse crossbar concentrators [7], [9] whose performance with respect to crosspoint complexity matches the previously reported sparse crossbar concentrators while offering regular fanin and fanout for any number of inputs and outputs. The crux of these new concentrator designs is a transformation theorem that can be applied to any sparse crossbar concentrator to convert it into another sparse crossbar concentrator. Indeed, all the new sparse crossbar concentrators given in the paper are obtained by transforming fat-and-slim crossbars using this theorem. ${ }^{1}$ We also describe an efficient algorithm to route arbitrary concentration assignments on these new sparse crossbars.

\section{Preliminary Facts}

An $(n, m, c)$-sparse crossbar concentrator is a bipartite graph $G=$ $(I, O, E)$, with a set of $n$ inputs $(I)$, a set of $m$ outputs $(O)$, and a set of edges $(E)$ such that there exist a matching between any $c$ or fewer inputs and an equal number of outputs, where $c$ is called its capacity. $\mathcal{G}$ is called a full capacity concentrator when $c=m$, and it is called a bounded capacity concentrator, otherwise. The edges in $E$ are called the crosspoints of $\mathcal{G}$. The number of crosspoints of $\mathcal{G}$ gives its crosspoint complexity. The number of outputs (inputs) to

1. Other implications of this transformation theorem can be found in [3].

- The authors are with the Electrical Engineering Department, University of Maryland at College Park, College Park, MD 20742.

E-mail: guo@eng.umd.edu.

Manuscript received 19 Feb. 1996.

For information on obtaining reprints of this article, please send e-mail to: tc@computer.org, and reference IEEECS Log Number 105710. 
which an input (output) is connected is called its fanout (fanin), and the maximum number of outputs (inputs) to which an input (output) in $G$ is connected is called the fanout (fanin) of $G$. A sparse crossbar is called regular if both the fanouts of its inputs and the fanins of its outputs differ by no more than two.

The set of outputs (inputs) which are connected to a set of inputs (outputs) $X$ is called the neighbor set of $X$, and denoted $N(X)$. The set of outputs (inputs) which are not connected to a set of inputs (outputs) $Y$ is called the null set of $Y$, and denoted $\Phi(Y)$.

The direct sum $G_{1}+G_{2}$ of sparse crossbars $G_{1}=\left(I_{1}, O, E_{1}\right)$ and $G_{2}=\left(I_{2}, O, E_{2}\right)$ is another sparse crossbar given by $G_{1}+G_{2}=\left(I_{1} \cup\right.$ $\left.I_{2}, O, E_{1} \cup E_{2}\right)$.

An $(n, m)$-sparse crossbar $G=(I, O)$ is called a fat-and-slim crossbar if any $n-m$ of its $n$ inputs are connected to all the outputs in $O$, and each of the remaining $m$ inputs is connected to a distinct output. For notational convenience, we will represent an $(n, m)$-fatand-slim crossbar $G$ by an $m \times n$ adjacency (binary) matrix, $A_{G}=$ $\left[a_{i, j}\right]_{m \times n}$, where a " 1 " entry in column $i$ and row $j$ represents a crosspoint between input $i$ and output $j$. An $(n, m)$-fat-and-slim crossbar then has the adjacency matrix $\left[J_{m, n-m} \mid I_{m}\right]$, where $J_{m, n-m}$ denotes a $m \times(n-m)$ matrix of " 1 "s, and $I_{m}$ denotes the $m \times m$ identity matrix. ${ }^{2}$

We will need the following well-known theorem to prove the main results in the next two sections.

HALL'S THEOREM. Let $A$ be a finite set and $A_{1}, A_{2}, \ldots A_{n}$ be arbitrary subsets of $A$. There exist distinct elements $a_{i} \in A_{i}, 1 \leq i \leq n$, iff the union of any $j$ of $A_{1}, A_{2}, \ldots A_{n}$ contains at least $j$ elements, $1 \leq j \leq n$.

\section{Full Capacity Regular Sparse Crossbars}

We begin with the following restatement of the fat-and-slim crossbar construction introduced in [9], when $n=p m$ for some positive integer $p$.

COROLlaRY 3.1. Let $G=(I, O)$ be a $(p m, m)$-sparse crossbar. Let $O=$ $\{1,2, \ldots, m\}$, and suppose $I$ is partitioned into $p$ sets: $I_{1}, I_{2}, \ldots, \ldots, I_{p}$ where $I_{k}=\{(k, 1),(k, 2), \ldots,(k, m)\}$. Suppose that every input in set $I_{k}, 1 \leq k \leq p-1$ is connected to all outputs in $O$ and input $j$ of set $I_{p}(1 \leq j \leq m)$ is connected to output $j$. Then, $G$ is an optimal $(p m, m)$-concentrator.

A regular fat-and-slim crossbar is constructed by rearranging the adjacency matrix of a fat-and-slim crossbar. More specifically, let row $i$ of $A_{G}$ be divided into $p$ sections:

$$
S_{i, j}=\left(a_{i, j m-m+1}, a_{i, j m-m+2}, \ldots, a_{i, j m}\right), 1 \leq j \leq p, 1 \leq i \leq m .
$$

It is easy to see that $S_{i, j}, 1 \leq j \leq p-1$ consists of $m$ " 1 "s, and $S_{i, p}$ consists of only one " 1 " entry, $1 \leq i \leq m$. Furthermore, the following lemma holds.

LEMMA 3.1. Let $G=(I, O)$ be a $(p m, m)$-fat-and-slim crossbar, and let $Y$ be any subset of $r \leq m$ rows in the adjacency matrix $A_{G}$, of $G$. Suppose that, for each $y \in Y$, entries in the columns in $S_{y, p}$ of $A_{G}$ are exchanged with entries in the columns in $S_{y, \alpha}$ without altering their relative order, where $1 \leq \alpha \leq p-1$, and let this new matrix be denoted by $A_{\widetilde{\mathcal{G}}}$. Then the sparse crossbar $\tilde{\mathcal{G}}$ that corresponds to matrix $A_{\widetilde{\mathcal{G}}}$ is an optimal $(\mathrm{pm}, \mathrm{m})$-concentrator.

PROOF. Obviously, the crosspoint complexity of $\mathcal{G}$ has not been affected by the transformation given in the lemma, so that

2. The words "row" and "column" will be used interchangeably with words "output" and "input," respectively.
$\widetilde{\mathcal{G}}$ has the same crosspoint complexity as $\mathcal{G}$, and is, therefore, optimal. Hence, we only need to show that $\widetilde{\mathcal{G}}$ is a concentrator. Let $A_{\widetilde{G}}$ be partitioned into $p m \times m$-matrices so that $A_{\widetilde{G}}=\left[\tilde{A}_{1}\left|\tilde{A}_{2}\right| \ldots \mid \tilde{A}_{p}\right]$, where $\tilde{A}_{i}$ is an $m \times m$ matrix, $1 \leq i \leq p$. The following two properties are easily verified.

PROPERTY 1 . The diagonal of $\tilde{A}_{i}, 1 \leq i \leq p$ consists of " 1 " entries.

PROPERTY 2. The null set of any row of $\tilde{\mathcal{G}}$ is a subset of the columns in $\tilde{A}_{i}$ for some $j, 1 \leq j \leq p$.

Now, let $X$ be any subset of $k \leq m$ columns. If $X \subseteq I_{j}$ for any $j$, $1 \leq j \leq p$, then, by Property $1,|N(X)| \geq k$. Suppose $X \nsubseteq I_{j}$, for all $j, 1 \leq j \leq p$, then there must exist columns $x_{q}, x_{r} \in X$ such that $x_{q} \in I_{q}$ and $x_{r} \in I_{r}$, where $1 \leq r \neq q \leq p$. Furthermore, we must have $\left|N\left(\left\{x_{q}, x_{r}\right\}\right)\right|=m$, since, if a row $y$ is not in the neighbor set of either $x_{q}$ or $x_{r}$, then $x_{q}$ and $x_{r}$ must both belong to $\Phi(y)$, i.e., the null set of $y$, contradicting Property 2 . It follows that the union of the neighbor sets of any $k$ inputs of $\tilde{\mathcal{G}}$ must have at least $k$ outputs and, by Hall's theorem, $\widetilde{\mathcal{G}}$ is a concentrator.

We use this result to obtain a regular $(p m, m)$-fat-and-slim crossbar.

THEOREM 3.1. Let $\mathcal{G}=(I, O)$ be a $(p m, m)$-fat-slim-crossbar, $\gamma=\lfloor m / p\rfloor$, $\beta=m-\gamma \times p$, and let $S_{i, j}, 1 \leq i \leq m, 1 \leq j \leq p$ be defined, as in (1). Suppose $\tilde{\mathcal{G}}=(I, O)$ is a $(\mathrm{pm}, \mathrm{m})$-sparse crossbar obtained by modifying the adjacency matrix $A_{G}$ of $G$ as follows:

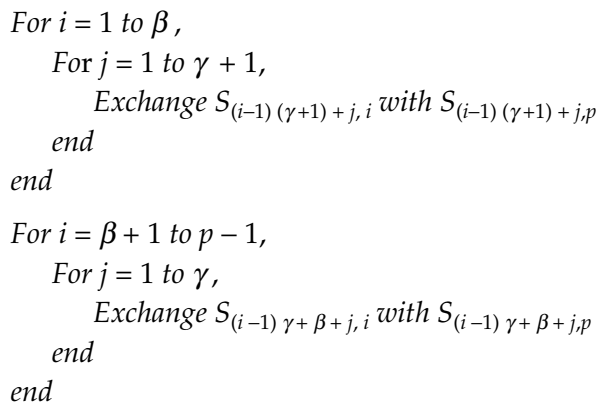

Then, $\tilde{\mathcal{G}}=(I, O)$ is an optimal regular $(p m, m)$-concentrator with a minimum crosspoint complexity.

PROOF. From Theorem 3.1, we know that $\widetilde{\mathcal{G}}$ has a minimum number of crosspoints, and all of its outputs have the same fanin. Furthermore, the construction given in the theorem moves $\gamma+1$ rows from the submatrix $A_{p}$ into each of the submatrices $A_{1}, A_{2}, \ldots, A_{\beta}$ and $\gamma$ rows into each of the submatrices $A_{\beta+1}$, $A_{\beta+2}, \ldots, A_{p-1}$. Therefore, the fanout of the inputs in set $I_{i}, 1 \leq i$ $\leq \beta$ is either $m-\gamma-1$ or $m-\gamma$, and the fanout of the inputs in set $I_{j}, \beta+1 \leq j \leq p$ is either $m-\gamma$ or $m-\gamma+1$. It follows from Lemma 3.1 that $\tilde{\mathcal{G}}$ is a regular $(p m, m)$-concentrator with a minimum number of crosspoints.

We illustrate this construction in Fig. 1, for $n=15, m=5, p=3$, and $\gamma=1$. It is seen that the fanout of any input that belongs to $I_{1}$ or $I_{2}$ is either 3 or 4 , and the fanout of any input that belongs to $I_{3}$ is either 4 or 5 . 


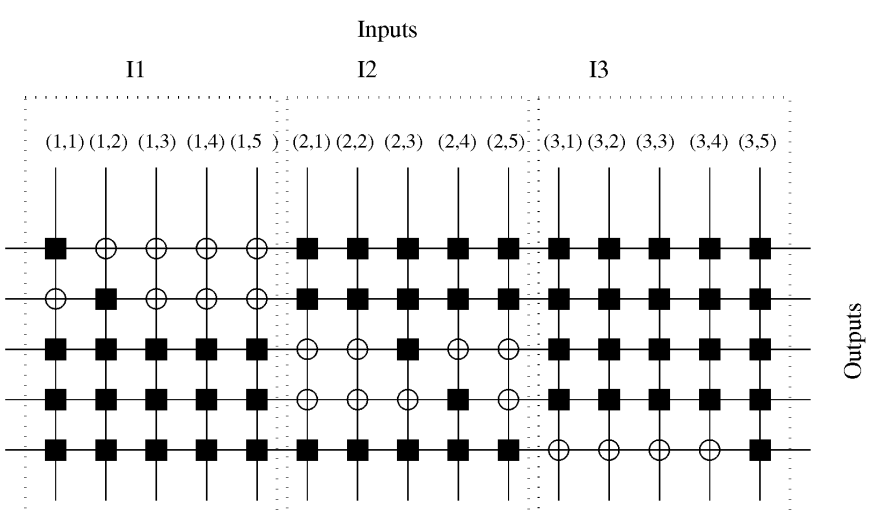

Fig. 1. A regular $(15,5)$-concentrator.

We have just given a construction for a family of optimal regular $(p m, m)$-sparse crossbar concentrators. In the following, we extend this construction to any number of inputs and outputs. As we will see, the basis for this extension is a powerful result (Theorem 3.2 and Corollary 3.2) that can be used to balance the fanout in many sparse crossbar concentrators.

We first state the following lemma, which is a direct consequence of Hall's theorem.

LEMMA 3.2. Any c inputs of an $(n, m)$-sparse crossbar network are connected to at least $c$ outputs if and only if the adjacency matrix of $G$ does not contain an $(m-k+1) \times k$ zero submatrix for all $k ; 1 \leq k \leq c$.

Let $A_{G}$ be the adjacency matrix of an $(n, m)$-sparse crossbar network $G$ and let $x$ and $y$ be any two columns in $A_{G}$. We say that column $x$ covers column $y$ if $N(y) \subseteq N(x)$.

THEOREM 3.2. Let $x$ and $y$ be any two columns in the adjacency matrix of an $(n, m)$-sparse crossbar $G$, where $x$ covers $y$. Let $a_{i_{1}, x}, a_{i_{2}, x}, \ldots, a_{i_{r}, x}$ be a block of $r$ " 1 "s in $x$, and let $B$ be a matrix obtained by exchanging $a_{i_{l}, x}$ with $a_{i_{l}, y}, 1 \leq l \leq r$. If $A_{G}$ does not have an $(m-k+1) \times k$ zero submatrix, where $1 \leq k \leq n$, then neither does $B$.

PROOF. Suppose $A_{G}$ does not have an $(m-k+1) \times k$ zero submatrix but $B$ does.

1) If the $k$ columns in the zero submatrix include neither column $x$ nor $y$, then, obviously, $A_{G}$ should also contain the same $(m-k+1) \times k$ zero submatrix, a contradiction.

2 ) If the $k$ columns in the zero submatrix in $B$ include column $y$ but not $x$, then, since every row in column $y$ of $B$ contains a " 1 " whenever the corresponding row in column $y$ of $A_{G}$ contains a " 1 ," and column $x$ is not included in the zero matrix, $A_{G}$ should also contain the same ( $m-$ $k+1) \times k$ zero submatrix, a contradiction.

3) If the $k$ columns in the zero submatrix include column $x$ but not $y$, then, since column $x$ of $B$ covers column $y$ of $A_{G}$ exchanging column $x$ with $y$ in $A_{G}$ shows that $A_{G}$ must contain an $(m-k+1) \times k$ zero submatrix, a contradiction.

4) If the $k$ columns in the zero submatrix include both column $x$ and $y$, then the zero submatrix in $B$ can only include the unchanged rows of column $x$ and $y$. Therefore, $A_{G}$ should also have the same $(m-k+1) \times k$ zero submatrix, a contradiction.

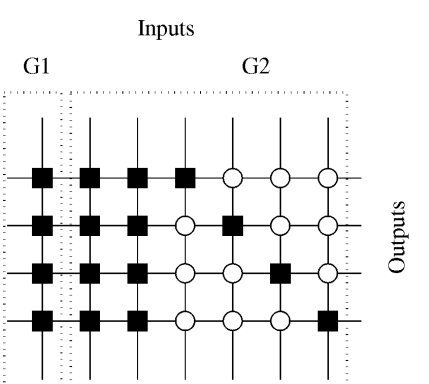

(a) Graph G

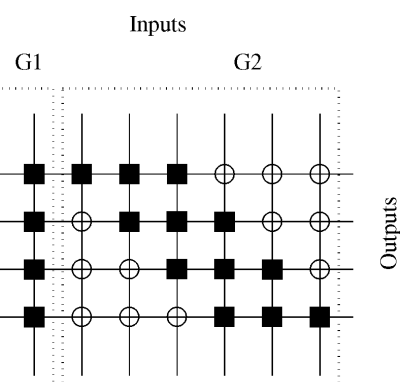

(b) Graph G after it is transformed into a partially banded sparse crossbar

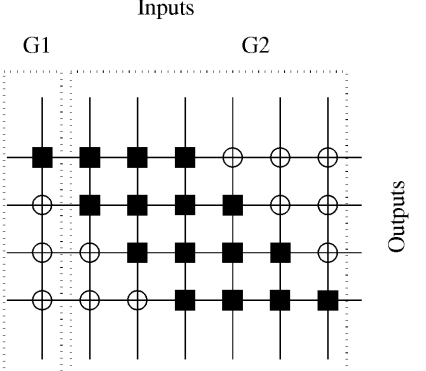

(c) Graph G is transformed into a banded sparse crossbar

Fig. 2. Illustration of the induction step in Theorem 3.3.

The lemma follows.

The following is a direct corollary of Theorem 3.2 and Lemma 3.2. COROLLARY 3.2. Let $G$ be a sparse crossbar concentrator with capacity $c \leq m$ and two inputs $x$ and $y$, where $x$ covers $y$. If a subset of " 1 " entries in $x$ are exchanged with the corresponding rows in $y$, then the resulting sparse crossbar is also a concentrator with capacity $c$.

Furthermore, we can use this result to obtain a banded sparse crossbar concentrator.

DEFINITION. An $(n, m)$-sparse crossbar $\mathcal{G}$ is called banded if its adja-

$$
\begin{aligned}
\text { cency matrix } A_{G}=\left[a_{i, j}\right] \text { is given by } \\
\qquad a_{i, j}= \begin{cases}1 & \text { if } i \leq j \leq i+n-m \\
0 & \text { if } j<i \text { or } j>i-n+m\end{cases}
\end{aligned}
$$

for $i=1,2, \ldots, m$.

THEOREM 3.3 Every banded $(n, m)$-sparse crossbar is an optimal $(n, m)$ concentrator.

PROOF. It follows from Corollary 3.2.

We will now use a banded sparse crossbar concentrator to obtain an optimal regular $(n, m)$-sparse crossbar concentrator for any positive integers $n$ and $m \leq n$.

Let

$$
\alpha= \begin{cases}\left\lfloor\frac{(n-m+1) m}{n}\right\rfloor & \text { if } n<\frac{3 m}{2} \\ \left\{\frac{(n-m+1) m}{n}\right] & \text { if } \frac{(n-m+1) m}{n}-\left\lfloor\frac{(n-m+1) m}{n}\right\rfloor<0.5, \text { and } n \geq \frac{3 m}{2} \\ \left\lceil\frac{(n-m+1) m}{n}\right] & \text { if } \frac{(n-m+1) m}{n}-\left\lfloor\frac{(n-m+1) m}{n}\right\rfloor \geq 0.5, \text { and } n \geq \frac{3 m}{2}\end{cases}
$$

$\beta=n-m-\alpha+1$

Let $G_{1}$ be a $(\beta, m)$-full crossbar and $G_{2}$ be an $(m+\alpha-1, m)$ banded crossbar concentrator Let $G=G_{1}+G_{2}$. It can be verified that $G$ is an $(n, m)$-sparse crossbar concentrator whose adjacency matrix $A_{G}=\left[a_{i, j}\right]$ is given by 
Inputs

$\mathbf{J}$

$\mathrm{U}$

B

L

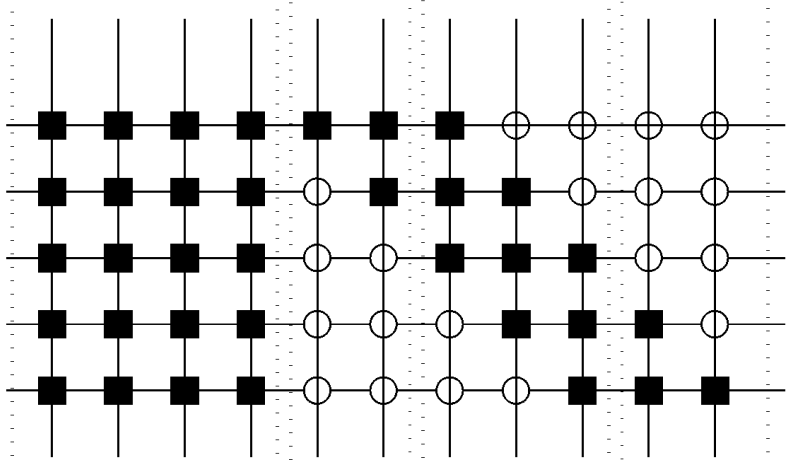

Fig. 3. An $(11,5)$-concentrator decomposed into four sections.

$$
a_{i, j}= \begin{cases}1 & \text { if } i+\beta \leq j \leq i+n-m+\beta \\ 1 & \text { if } 1 \leq j \leq \beta \\ 0 & \text { if } \beta<j<i+\beta \text { or } j>i+n-m+\beta\end{cases}
$$

for $i=1,2, \ldots, m$.

$A_{G}$ can be decomposed as $A_{G}=\left[J_{m \times \beta}\left|U_{m \times(\alpha-1)}\right| B_{m \times(n-\beta-2 \alpha+2)} \mid\right.$ $\left.L_{m \times(\alpha-1)}\right]$, where

$J_{m \times \beta}$ is a matrix of " 1 "s,

$U_{m \times(\alpha-1)}$ is an upper triangular matrix,

$B_{m \times(n-\beta-2 \alpha+2)}$ is a banded matrix,

$L_{m \times(\alpha-1)}$ is a lower triangular matrix.

Fig. 3 shows the decomposition of a sparse crossbar concentrator.

LEMMA 3.3. Let $H=[J|U| L]$ where $J, U, L$ are matrices described above. If $n \geq \frac{3 m}{2}$, then matrix $H$ can be rearranged using the column exchange operation described in Theorem 3.2 to obtain a matrix $\tilde{H}$, in which every column has either $\alpha \pm 1$ or $\alpha$ " 1 "s.

PROOF. See the Appendix.

We now have our main theorem.

THEOREM 3.4. For any positive integer $n$ and $m \leq n$, the $(n, m)$-fat-andslim sparse crossbar concentrator can be rearranged to obtain a sparse crossbar concentrator with fanout of either $\alpha \pm 1$ or $\alpha$, fanin of $n-m+1$ and minimum number of crosspoints, where $\alpha$ is defined by (3).

PROOF. Let $A_{G}=\left[J_{m \times \beta}\left|U_{m \times(\alpha-1)}\right| B_{m \times n-\beta-2 \alpha+2} \mid L_{m \times(\alpha-1)}\right]$, where $J, U, B, L$ are defined as before. We already established that a fat-andslim crossbar can be expressed as a direct sum of a full crossbar and banded sparse crossbar, and that if $n \geq 3 m / 2$, then the columns of this direct sum can be balanced to have $\alpha$ or $\alpha \pm 1$ crosspoints each without altering its concentration property. It remains to be shown that the statement also holds when $n<\frac{3 m}{2}$. In this case, suppose that we assign $\alpha$ "1"s to each column in $B$ and $(\beta+2 \alpha-2)(\alpha+1)$ " 1 "s to the columns in $H$, where $H$ is the direct sum of $J, U$, and $L$ as before. Then the number of " 1 "s which are left unassigned is given by

$$
\gamma=(n-m+1) m-\alpha n-(\beta+2 \alpha-2),
$$

Since $(n-m+1) m-\alpha n \geq 0$, we must have $\gamma \geq-(\beta+2 \alpha-2)$. Thus, if $\gamma \leq 0$, then the average number of " 1 "s in $H$ must
Inputs

U

B

L

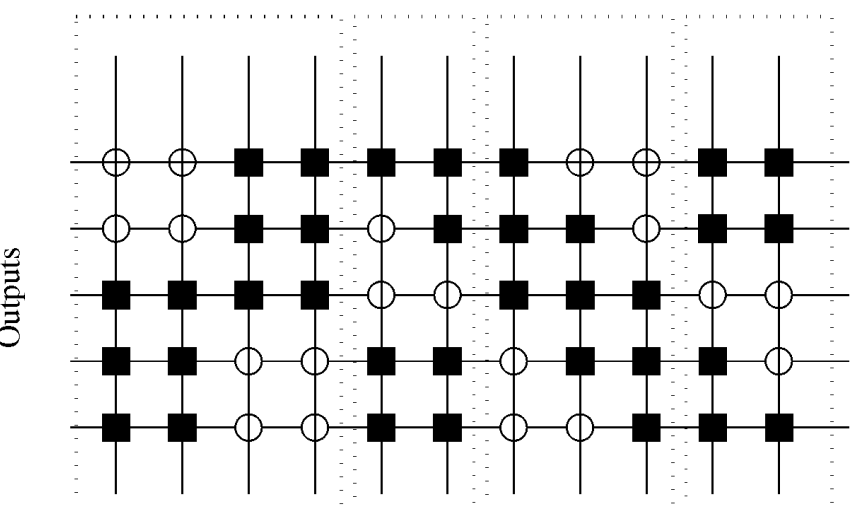

冚

Fig. 4. The $(11,5)$-concentrator in Fig. 3 after it is balanced.

obviously be in the region of $[\alpha, \alpha+1]$. Therefore, one can use the same procedure described in the proof of Lemma 3.3 to balance the columns in $H$ so that each of its columns has either $\alpha$ or $\alpha+1$ " 1 "s. On the other hand, if $\gamma>0$, then the average number of " 1 "s over the columns in $H$ is more than $\alpha+1$. In this case, we can preserve $\left\lfloor\frac{\gamma}{m-(\alpha+1)}\right\rfloor$ columns of " 1 "s in $J$, and balance the remaining columns in $J$ with the columns in $U$ and $L$ so that each of the balanced columns has $\alpha+1$ " 1 "s. Now, the inequality

$$
0 \leq(n-m+1) m-\alpha n \leq n,
$$

together with (5), implies $\gamma \leq n-(\beta+2 \alpha-2)$, where $n-(\beta+$ $2 \alpha-2)$ is the number of columns in $B$. Therefore, one can distribute the extra $\gamma$ "1"s from the unbalanced columns in $J$ to the columns in $B$ with each having at most one additional "1." The proof follows.

Fig. 4 shows how the crosspoints in the sparse crossbar of Fig. 3 are balanced by distributing the " 1 "s in $J$ into $U$ and $L$, when $n<$ $3 m / 2$. In this case, $\gamma=-6<0$ so that crosspoints are balanced without splitting $J$ into two parts, as outlined in the proof.

\section{ROUTING}

Routing a concentrator amounts to activating some of its $k$ switches such that a desired set of $k, 1 \leq k \leq c$ inputs can be connected to some $k$ distinct outputs by disjoint paths, where $c$ is the capacity of the concentrator. In the following, we present a routing algorithm for an optimal regular $(\mathrm{pm}, \mathrm{m})$-sparse crossbar concentrator, where $p$ and $m / p$ are positive integers. Algorithms for other values $p$ and $m$ can be obtained by modifying the algorithm presented here.

LEMMA 4.1 Any $m$ inputs of an (n, m)-full crossbar can be routed to $m$ outputs in $\mathrm{O}(\log n)$ time on a binary tree of $n$ processors and $\mathrm{O}(n)$ time on a single processor.

PROOF. See [3].

Let $G=(I, O)$ be an optimal regular $(p m, m)$-sparse crossbar concentrator, as defined in Theorem 3.1, where $p$ and $m / p$ are integers, $O=\{1,2, \ldots, m\}$, and $I$ is partitioned into $p$ sets, $I_{1}, I_{2}, \ldots, \ldots$, $I_{p}$, where $I_{i}=\{(i, 1),(i, 2), \ldots,(i, m / p)\}$.

Let $N_{i, j}$ be the neighbor set of input $(i, j) \in I_{i}$,

$$
V_{i}=\left\{N_{i, 1} \cap N_{i, 2} \cap \ldots \cap N_{i, \frac{m}{p}}\right\},
$$




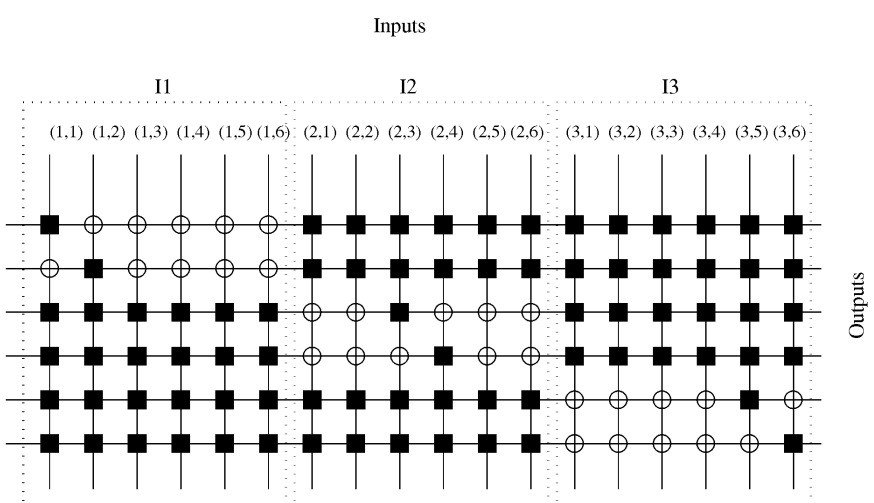

Fig. 5. $(18,6)$-concentrator.

$U_{i}=O \backslash V_{i}$, and $W_{i}$ be the inputs in $I_{i}$ that are connected to at least one output in $U_{i}$. In Fig. $5, U_{1}=\{1,2\}, V_{1}=\{3,4,5,6\}$, and $W_{1}=\{(1,1)$, $(1,2)\}$.

From the procedure described in Theorem 3.1, one can see that $\left|U_{i}\right|=m / p,\left|W_{i}\right|=m / p$, and $\left|V_{i}\right|=m-m / p$. Furthermore, all the inputs in set $I_{i}$ are connected to outputs in set $V_{i}$ by a full crossbar, and every input in $W_{i}$ is connected to a distinct output in set $U_{i}$. We also note that $U_{i} \cap U_{j}=\varnothing, 1 \leq i \neq j \leq p$, and $\sum_{i=1}^{p}\left|U_{i}\right|=m$.

Let $R$ be a subset of $k \leq m$ inputs that request to be routed to $k$ outputs. We select the first $m-k$ unused inputs in set $I_{1}$ and combine them with set $R$ to form a new set of inputs $\widetilde{R}$, where $|\widetilde{R}|=m$. We also let $R_{i}=\widetilde{R} \cap I_{i}, 1 \leq i \leq p$, and note that $\sum_{i=1}^{p} R_{i}=m$.

Algorithm 4.1. Let $R_{i}, U_{i}, 1 \leq i \leq p$, be defined as above.

1) For $i=1$ to $p$,

$$
\text { Let } a_{i}= \begin{cases}2 & \text { if }\left|R_{i}\right|>m-\frac{m}{p} \\ 1 & \text { if } \frac{m}{p} \leq\left|R_{i}\right| \leq m-\frac{m}{p} \\ 0 & \text { if }\left|R_{i}\right|<\frac{m}{p}\end{cases}
$$

2) If $a_{j}=2$, for some $j, 1 \leq j \leq p$ then

2.1) Assign inputs in set $R_{j} \cap W_{j}$ to outputs in set $U_{j}$.

2.2) Assign all the inputs in sets $R_{i}(i \neq j)$ to the unassigned outputs in $U_{j}$ using Lemma 4.1.

2.3) Assign the remaining inputs in $R_{j}$ to the rest of the $m-$ $m / p$ outputs in $V_{j}$ by assigning input $(j, l)$ to output $l$, where $l \in[1, m]$.

3) Else, let (for $1 \leq i \leq p)$

3.1)

$$
b_{i}= \begin{cases}0 & \text { if }\left|R_{i}\right| \geq \frac{m}{p} \\ 1 & \text { if }\left|R_{i}\right|<\frac{m}{p}\end{cases}
$$

$$
r_{i}=\sum_{j=1}^{i} a_{j}, s_{i}=\sum_{j=1}^{i} b_{j}
$$

\begin{tabular}{|c|c|c|c|c|c|c|}
\hline R1 & $\mathrm{R} 2$ & R3 & \multicolumn{2}{|l|}{ R4 } & \multicolumn{2}{|c|}{ R5 } \\
\hline U1 & U2 & U3 & & $\mathrm{U}_{4}$ & & U5 \\
\hline & & (a) & & & & \\
\hline$R^{\prime} 1$ & & & $R^{\prime} 3$ & & $R^{\prime} 4$ & $R^{\prime} 5$ \\
\hline $\mathrm{U}^{\prime} 1$ & $U^{\prime}$ & $\mathrm{U}^{\prime}$ & & $\mathrm{U}^{\prime}$ & & $U^{\prime} 5$ \\
\hline
\end{tabular}

$$
\begin{gathered}
d_{i}= \begin{cases}r_{i} & \text { if } a_{i}=1 \\
s_{i}+r_{p} & \text { if } a_{i}=0\end{cases} \\
R_{d_{i}}^{\prime}=R_{i}, U_{d_{i}}^{\prime}=U_{i}
\end{gathered}
$$

(b)

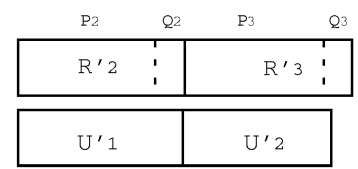

(c)
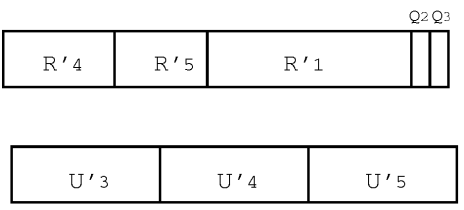

(d)

Fig. 6. Illustration of routing algorithm.

3.4) Let $P_{i}$ be the subset of the first $\frac{m}{p}$ inputs in $R_{i}^{\prime}, 2 \leq i \leq$ $r_{p}$, and let $Q_{i}=R_{i}^{\prime} \backslash P_{i}$. Assign the inputs in $P_{i}$ to $U_{i-1}^{\prime}$, $2 \leq i \leq r_{p}$

3.5) Assign the inputs in $R_{r_{p}+1}^{\prime}, R_{r_{p}+2}^{\prime}, \ldots, R_{p}^{\prime}, R_{1}^{\prime}, Q_{2}, Q_{3}, \ldots, Q_{r_{p}}$ to $U_{r_{p}}^{\prime}, U_{r_{p}+1}^{\prime}, \ldots, U_{p}^{\prime}$.

THEOREM 4.1. Any $k$ inputs of an optimal regular (pm, $m)$-concentrator can be assigned to the $k$ outputs in $\mathrm{O}(\log n)$ time on a binary tree of $n$ processors, and $\mathrm{O}(n)$ time on a single processor by Algorithm 4.1, where $n=p m$.

PROOF. See [3].

It follows that the routing time of an optimal regular sparse crossbar concentrator matches the time complexity of routing a full crossbar.

We use Fig. 5 to illustrate Step 2 of this algorithm. Let the requested inputs be $(1,1),(1,3),(1,4),(1,5),(1,6),(2,4)$. So $\left|R_{1}\right|=5$, $\left|R_{2}\right|=1$, and $\left|R_{3}\right|=0$. Since $\left|R_{1}\right|>m-m / p=6-2=4$, the algorithm will run Step 2 as follows:

1.1) Assign input $(1,1)$ to output 1.

1.2) Assign input $(2,4)$ to output 2.

1.3) Assign inputs $(1,3),(1,4),(1,5),(1,6)$ to outputs $3,4,5,6$.

We use Fig. 6 to illustrate Step 3 of this algorithm.

The requests are partitioned into five sets $R_{1}, R_{2}, R_{3}, R_{4}, R_{5}$ as shown in Fig. 6a.

After Step 3.3, they are assigned new indexes $R_{1}^{\prime}, R_{2}^{\prime}, R_{3}^{\prime}, R_{4}^{\prime}, R_{5}^{\prime}$ as shown in Fig. 6b.

In Step 3.4, the inputs in $P_{2}$ are connected to outputs in $U_{1}^{\prime}$, and the inputs in $P_{3}$ are connected to outputs in $U_{2}^{\prime}$ as shown in Fig. 6c. In Step 3.5, inputs in $R_{4}^{\prime}, R_{5}^{\prime}, R_{1}^{\prime}, Q_{1}$, and $Q_{2}$ are connected to outputs in $U_{3}^{\prime}, U_{4}^{\prime}$, and $U_{5}^{\prime}$ as shown in Fig. $6 \mathrm{~d}$. 


\section{Concluding Remarks}

A possible extension of the results given in the paper would be to obtain bounded capacity regular sparse crossbar concentrators. In this case, no construction is known except for a few specific cases. What compounds this problem is the lack of a potentially tight explicit lower bound. Another aspect of sparse crossbar concentrators that has not been discussed here is their reliability. In the case of optimal sparse crossbar concentrators, such as those described in the paper, even a single switch failure will render them ineffective. On the other hand, one can view an optimal sparse crossbar as a full crossbar with faulty switches. In that case, the constructions given here can form a basis for investigating the reliability of full crossbars. This investigation and its results will be deferred to another place.

\section{APPENDIX}

\section{The Proof of Lemma 3.3}

We first note that the total number of " 1 "s in matrix $H$ is $(n-m+1)$ $m-\alpha(n-\beta-2 \alpha+2)$ and the number of inputs in $H$ is $\beta+2 \alpha-2$. So, the average number of " 1 "s in $H$ for each column is

$$
\begin{aligned}
& \frac{(n-m+1) m-\alpha(n-\beta-2 \alpha+2)}{\beta+2 \alpha-2} \\
= & \frac{(n-m+1) m-\alpha n}{\beta+2 \alpha-2}+\alpha \\
= & \begin{cases}\frac{(n-m+1) m-\lfloor(n-m+1) m / n\rfloor n}{\beta+2 \alpha-2}+\alpha & \text { if }(n-m+1) m / n-\lfloor(n-m+1) m / n\rfloor<0.5 \\
\frac{(n-m+1) m-[(n-m+1) m / n] n}{\beta+2 \alpha-2}+\alpha & \text { if }(n-m+1) m / n-\lfloor(n-m+1) m / n\rfloor \geq 0.5\end{cases} \\
= & \begin{cases}\frac{(n-m+1) m-\lfloor(n-m+1) m / n\rfloor n}{n-m+\lfloor(n-m+1) m / n\rfloor-1}+\alpha & \text { if }(n-m+1) m / n-\lfloor(n-m+1) m / n\rfloor<0.5 \\
\frac{(n-m+1) m-[(n-m+1) m / n] n}{n-m+[(n-m+1) m / n]-1}+\alpha & \text { if }(n-m+1) m / n-\lfloor(n-m+1) m / n\rfloor \geq 0.5 .\end{cases}
\end{aligned}
$$

We will show that the absolute value of the fractional term in each of the expressions is bounded by 1 . First, suppose

$$
0 \leq(n-m+1) m / n-\lfloor(n-m+1) m / n\rfloor<0.5 .
$$

It can be verified that, for $4 \leq n \leq 100$, and $2 \leq m \leq 2 n / 3$

$$
0 \leq \frac{(n-m+1) m-\lfloor(n-m+1) m / n\rfloor n}{n-m+\lfloor(n-m+1) m / n\rfloor-1} \leq 0.86 .
$$

For $n>100,(6)$ gives

$$
0 \leq \frac{(n-m+1) m-\lfloor(n-m+1) m / n\rfloor n}{n-m+\lfloor(n-m+1) m / n\rfloor-1}<\frac{0.5 n}{n-m+\lfloor(n-m+1) m / n\rfloor-1},
$$

or

$$
0 \leq \frac{(n-m+1) m-\lfloor(n-m+1) m / n\rfloor n}{n-m+\lfloor(n-m+1) m / n\rfloor-1}<\frac{0.5 n}{n-m+(n-m+1) m / n-2},
$$

or

$$
0 \leq \frac{(n-m+1) m-\lfloor(n-m+1) m / n\rfloor n}{n-m+\lfloor(n-m+1) m / n\rfloor-1}<\frac{0.5 n^{2}}{n^{2}-m^{2}+m-2 n},
$$

or

$$
0 \leq \frac{(n-m+1) m-\lfloor(n-m+1) m / n\rfloor n}{n-m+\lfloor(n-m+1) m / n\rfloor-1}<\frac{0.5}{1-\frac{m^{2}}{n^{2}}+\frac{m}{n^{2}}-\frac{2}{n}} .
$$

Since $n \geq \frac{3 m}{2}$, and $n>100$, we have $\frac{m}{n} \leq \frac{2}{3}$, and $\frac{1}{n}<0.01$, so that

$$
0 \leq \frac{(n-m+1) m-\lfloor(n-m+1) m / n\rfloor n}{n-m+\lfloor(n-m+1) m / n\rfloor-1}<\frac{0.5}{1-\frac{4}{9}-0.02}
$$

$$
0 \leq \frac{(n-m+1) m-\lfloor(n-m+1) m / n\rfloor n}{n-m+\lfloor(n-m+1) m / n\rfloor-1}<1 .
$$

Similarly, for

$$
(n-m+1) m / n-\lfloor(n-m+1) m / n\rfloor \geq 0.5,
$$

it can again be verified that

$$
-1<\frac{(n-m+1) m-\lceil(n-m+1) m / n\rceil n}{n-m+\lceil(n-m+1) m / n\rceil-1} \leq 0 .
$$

It follows that, when $n \geq \frac{3 m}{2}$, the average number of " 1 "s for each column in $H$ is in the region $[\alpha-1, \alpha+1]$. Moreover, since $U$ is an upper triangular matrix and $L$ is a lower triangular matrix, the number of " 1 "s in $U$ and $L$ can be balanced using the column exchange operation defined in Theorem 3.2 so that each column in $U$ and $L$ has $\lceil\alpha / 2\rceil$ (or $\lfloor\alpha / 2\rfloor)$ " 1 "s. Finally, since $J$ is a matrix of " 1 "s, each column in $J$ can be balanced to have $\alpha \pm 1$ or $\alpha$ " 1 "s by distributing $m-\alpha \pm 1$ of its "1"s over any $\lceil\alpha / 2\rceil$ (or $\lfloor\alpha / 2\rfloor$ ) subset of the $m-\alpha$ empty rows of $2(m-\alpha \pm 1) / \alpha$ columns in $U$ or $L$. using the column exchange operation defined in Theorem 3.2. It follows that matrix $\tilde{H}$ can be balanced so that each of its columns has $\alpha \pm 1$ or $\alpha$ " 1 "s.

\section{ACKNOWLEDGMENTS}

This work is supported in part by the U.S. National Science Foundation under Grant No. NCR-9405539.

\section{REFERENCES}

[1] N. Alon, "Eigenvalues and Expanders," Combinatorics, vol. 6, pp. 8396, 1986.

[2] L.A. Bassalygo, "Asymptotically Optimal Switching Circuits," Problems of Information Transactions, vol. 17, no. 3, pp. 206-211, 1981.

[3] W. Guo, "Design And Optimization of Switching Fabrics for ATM Networks and Parallel Computer Systems," doctoral dissertation, Dept. of Electrical Eng., Univ. of Maryland at College Park, Aug. 1996.

[4] C. Jan and A.Y. Oruç, "Fast Self-Routing Permutation Switching on an Asymptotically Minimum Cost Network," IEEE Trans. Computers, vol. 42, no. 12, pp. 1,469-1,479, Dec. 1993.

[5] G.M. Masson, "Binomial Switching Networks for Concentration and Distribution," IEEE Trans. Comm., vol. 25, pp. 873-883, Sept. 1977.

[6] G.A. Margulis, "Explicit Constructions of Concentrators," Problems of Information Transactions, pp. 325-332, 1973.

[7] S. Nakamura and G.M. Masson, "Lower Bounds on Crosspoint in Concentrators," IEEE Trans. Computers, vol. 31, no. 12, pp. 1,1731,178, Dec. 1982.

[8] M.V. Chien and A.Y. Oruç, "Adaptive Binary Sorting Schemes and Associated Interconnection Networks," IEEE Trans. Parallel and Distributed Systems, vol. 5, no. 6, pp. 561-571, June 1994.

[9] A.Y. Oruç and H.M. Huang, "New Results on Sparse Crossbar Concentrators," Proc. CISS, Princeton Univ., 1994.

[10] N. Pippenger, "Superconcentrators," SIAM J. Computers, vol. 6, no. 2, pp. 298-304, 1977. 